\title{
Spatial and space-time distribution of Plasmodium vivax and Plasmodium falciparum malaria in China, 2005-2014
}

Samuel H. Hundessa', Gail Williams', Shanshan Li ${ }^{1}$, Jinpeng Guo², Linping Chen'1, Wenyi Zhang ${ }^{2 *}$ and Yuming Guo ${ }^{1 *}$

\begin{abstract}
Background: Despite the declining burden of malaria in China, the disease remains a significant public health problem with periodic outbreaks and spatial variation across the country. A better understanding of the spatial and temporal characteristics of malaria is essential for consolidating the disease control and elimination programme. This study aims to understand the spatial and spatiotemporal distribution of Plasmodium vivax and Plasmodium falciparum malaria in China during 2005-2009.
\end{abstract}

Methods: Global Moran's / statistics was used to detect a spatial distribution of local P. falciparum and P. vivax malaria at the county level. Spatial and space-time scan statistics were applied to detect spatial and spatiotemporal clusters, respectively.

Results: Both P. vivax and P. falciparum malaria showed spatial autocorrelation. The most likely spatial cluster of $P$. vivax was detected in northern Anhui province between 2005 and 2009, and western Yunnan province between 2010 and 2014. For P. falciparum, the clusters included several counties of western Yunnan province from 2005 to 2011 , Guangxi from 2012 to 2013, and Anhui in 2014. The most likely space-time clusters of P. vivax malaria and P. falciparum malaria were detected in northern Anhui province and western Yunnan province, respectively, during 2005-2009.

Conclusion: The spatial and space-time cluster analysis identified high-risk areas and periods for both $P$. vivax and $P$. falciparum malaria. Both malaria types showed significant spatial and spatiotemporal variations. Contrary to P. vivax, the high-risk areas for P. falciparum malaria shifted from the west to the east of China. Further studies are required to examine the spatial changes in risk of malaria transmission and identify the underlying causes of elevated risk in the high-risk areas.

Keywords: Plasmodium falciparum, Plasmodium vivax, Malaria, Spatial clustering, Space-time clustering

\section{Background}

Malaria is a life-threatening infectious disease severely affecting vulnerable communities in tropical and subtropical regions where the environment is suitable for transmission [1, 2] Although malaria transmission appears to be declining worldwide as a result of control interventions [2, 3], the 2015 estimation indicates that

\footnotetext{
*Correspondence: zwy0419@126.com; y.guo1@uq.edu.au

${ }^{1}$ Division of Epidemiology and Biostatistics, School of Public Health, University of Queensland, Herston Rd, Herston, QLD 4006, Australia

${ }^{2}$ Institute for Disease Control and Prevention, Academy of Military

Medical Science, Beijing, People's Republic of China
}

there are 214 million cases and 438,000 malaria deaths [4]. Malaria is caused by five species of Plasmodium: Plasmodium falciparum, Plasmodium vivax, Plasmodium ovale, Plasmodium malariae and Plasmodium knowlesi [1]. In China, P. vivax and P. falciparum are the main malaria parasites, with the former being the most dominant species [5]. Anopheles sinensis, Anopheles minimus, Anopheles dirus, and Anopheles lesteri are common malaria vectors in China [6].

Prior to 1949 , the annual number of malaria cases in China was estimated to be 30 million. Owing to its substantial public health importance, a Malaria Control 
Programme was initiated in 1955 [7]. Since then, the malaria burden has greatly declined $[8,9]$, but it has remained a serious public health problem in China with periodic outbreaks [10]. Following an epidemic peak in 2006 [11], control efforts were consolidated with the formulation of the National Malaria Control Programme (NMCP) in 2006 [12]. Overall, malaria cases have sharply declined with only 14,491 malaria cases reported in 2009 [13]. The National Malaria Elimination Programme (NMEP) was launched in 2010 [12]. Since then, substantial progress has been made. $P$. vivax malaria cases were reduced by $57.7 \%$ in one year [14], followed by a decline in geographical coverage [5]. However, P. falciparum greatly increased dominating the overall confirmed malaria cases since 2007. The proportion of $P$. falciparum malaria increased from $7.1 \%$ in 2009 [13] to $71.2 \%$ in 2013 [15]. Areas affected by P. falciparum have consistently increased from 17 provinces in 2006 to 20 in 2010, 22 in 2011 [14], and to 30 provinces in 2013 [14-16], involving formerly non-endemic provinces [17].

The distribution of malaria in China shows considerable variation at fine spatial resolution such as county [18, 19]. A better understanding of the spatiotemporal change in disease distribution is crucial for improving control interventions and health resource allocation. Several studies have used the spatial and space-time scan statistics to detect clustering of malaria [18-22] and other public health problems [23] in space and time. These techniques detect disease clusters while adjusting for varying population size among spatial and temporal scales under study. In China, these have been used to identify high-risk areas and periods of malaria in some endemic provinces [18-22, 24, 25]. However, few studies have analysed the spatial and space-time distribution of both $P$. vivax and $P$. falciparum malaria at the national level. The purpose of the present study is to fill this gap in the understanding of the spatial and spatiotemporal distribution malaria in China during 2005-2014.

\section{Methods}

The study was conducted in mainland China, which encompass 31 provinces/autonomous region/municipalities, and more than two thousand county-level divisions. According to the sixth national census in 2010, mainland China has a population of 1.3 billion [26].

\section{Data sources and its management}

Malaria case data between 2005 and 2014 were obtained from the China Information System for Disease Control and Prevention (CISDCP). Malaria is a notifiable disease in China. The laboratory-confirmed and suspected cases are reported to the county-level CDC within one day through an online infectious disease reporting system in use since 2004 [27]. Case investigation and identification are conducted within three days of receiving the case report. Cases are identified according to national standard criteria issued by the Chinese Ministry of Health [28]. Malaria case data includes the county name and associated county code (i.e., identification number), demographic information, date of diagnosis and travel history. These counties are the living addresses for malaria cases. The surveillance-response system covers the whole country which enabled us to conduct a national level study.

Malaria case data, population, and geo-coordinates of each county were linked via county code in R Software version 3.2.2 [29]. As input for the clustering analysis in SaTSan software (version 8.0), a coordinate file, case file and population files were separately generated in text format. To indicate locations of the clusters, the output of the clustering analysis was mapped in ArcGIS software version 10.3.1 [30].

\section{Spatial autocorrelation analysis}

A descriptive analysis was performed to explore countylevel annual trends of $P$. falciparum and $P$. vivax malaria cases in China during 2005-2014, and the results were plotted to reveal the annual trend. County level counts of $P$. vivax and $P$. falciparum malaria case for each year were used to test for the spatial distribution of the malaria in China. To explore the overall spatial autocorrelation within-country level local malaria cases, Global Moran's I statistics was applied in ArcGIS software version 10.3.1 [30]. Row standardization of spatial weight was allowed as criteria for identifying neighbouring counties [31]. If counties share a boundary the spatial weight element was assumed to be 1 ; otherwise it was assumed to be 0 . Based on this assumption, Global Moran's $I$ values were calculated for $P$. falciparum and $P$. vivax malaria separately. The value varies between -1.0 and +1.0 , where a value close to +1 indicates a tendency for spatial clustering (positive spatial autocorrelation) of areas with similar numbers of malaria cases, whereas -1.0 and 0.0 respectively indicate spatial dispersion (negative spatial autocorrelation) and a random spatial pattern. Global Moran's $I$ tests the null hypothesis that the distribution of malaria in China is random in space [32]. Significance $(\mathrm{p}<0.05)$ of the test statistic suggests that malaria cases are spatially clustered or dispersed. Following this, spatial scan statistics was used to identify locations of the significant cluster (i.e., high-risk areas) [33].

\section{Spatial and space-time cluster analysis}

In this study, two spatial scan statistics were performed: purely spatial cluster analysis and mixed space-time cluster analysis. The purely spatial cluster analysis (with the Poisson model) was performed for $P$. vivax and $P$. 
falciparum malaria separately in SaTScan Software. These techniques impose circular windows of different sizes to scan for spatial clustering. Kuldorff's spatial cluster detection statistics [34], was used to scan for counties with high rates of malaria. The maximum spatial cluster size was set to the default of $50 \%$ of the population at risk in the circular spatial window [18]. In addition to avoiding pre-selection bias, this enables scanning for clusters of different sizes [34]. The most likely and secondary clusters were not permitted to overlap. The space-time scan statistic was also performed with the Poisson model. The length of time aggregation was set to one year. The maximum temporal cluster sizes to be scanned were also set at the default of $50 \%$ of the populationat risk and $50 \%$ of the study period, respectively.

\section{Results}

\section{Overall trend of malaria}

A total of 247,540 malaria cases were reported in China during 2005-2014. An annual variation in number of malaria cases was observed with most cases in 2006 $(\mathrm{N}=73,283)$, comprising 56,258 P. vivax and 3113 P. falciparum malaria. Overall, P. vivax accounted for $75.2 \%$ of malaria cases, while $8.7 \%$ were $P$. falciparum. $16.2 \%$ of all malaria cases reported during this period were unidentified.

A substantial decrease in the yearly number of vivax malaria case was observed during the study period (Fig. 1a). The most rapid reduction in the number of $P$. vivax cases occurred after 2006. The number of areas with $P$. vivax cases declined from 791 counties in 2006 to 303 counties in 2014 with an overall reduction of $60.7 \%$. Numbers of $P$. falciparum malaria cases reached a peak in 2005, fell until 2008, then slightly increased, and have dominated since 2012 (Fig. 1a). Consequently, areas with P. falciparum greatly increased from 228 counties to 783 . Compared to 2005, areas with P. falciparum increased by more than $100 \%$.

\section{Sources of infection}

The data from 2005 to 2014 indicated 153, 051 local P. vivax and 19, 666 local P. falciparum malaria cases. Declining trends of local transmission were observed for both malaria types (Fig. 1b). Local P. vivax and P. falciparum malaria transmission declined by 99.79 and $98.42 \%$, respectively. The geographical distribution of the counties with local $P$. vivax and $P$. falciparum during this period was shown in Additional file 1.

In contrast to the decline in local malaria cases, an increasing trend was observed for imported malaria during this study period (Fig. 1c). Imported P. falciparum consistently increased from 2005 to 2014, reaching a peak in 2013. However, the imported $P$. vivax didn't show a significant change over the study period. Regarding the origin of infection, $P$. falciparum was mainly imported from Africa $(72.70 \%, \mathrm{~N}=7,700)$ and Myanmar $(17.7 \%$, $\mathrm{N}=1,878$ ) while the remaining cases were imported from Indonesia $(0.69 \%, \mathrm{~N}=73)$, Cambodia $(0.23 \%$, $\mathrm{N}=24)$, Laos $(0.19 \%, \mathrm{~N}=20)$, Pakistan $(0.13 \%, \mathrm{~N}=14)$, India $(0.09 \%, \mathrm{~N}=10)$, Papua New Guinea (PNG) $(0.06 \%$, $\mathrm{N}=6$ ), and Vietnam $(0.06 \%, \mathrm{~N}=6)$, and. $P$. vivax was mainly imported from Myanmar $(\mathrm{N}=5,613)$, Oceania and unspecified countries $(\mathrm{N}=1,171)$ (Additional file 2).

Spatial autocorrelation analysis of annual local malaria cases in China showed the existence of significant overall spatial autocorrelation each year during the study period. The tendency of spatial clustering was observed for both $P$. vivax and $P$. falciparum malaria (Table 1).

\section{Spatial clustering}

A purely spatial cluster analysis of annual $P$. vivax malaria indicated a non-random distribution of malaria cases in China during 2005-2014. The analysis detected significant spatial clusters (most likely and secondary) each year during the study period. The number of counties per most likely cluster ranged from 7 to 35 . The most likely cluster with the highest number of counties was detected in 2007 and that with the lowest number in 2013. The most likely spatial cluster of $P$. vivax included several counties in the Northern Anhui province from 2005 to 2009. Throughout the rest of the study period, the most likely cluster covered bordering counties along the western Yunnan province. The secondary clusters were also identified in many provinces, each year during the study period. Most of these clusters, especially those with a high relative risk were detected in Western Yunnan from 2005 to 2009 and in Northern Anhui until 2011 (Fig. 2). Overall, more than half of the local $P$. vivax cases were reported from the most likely and secondary cluster areas except in 2013, when the clusters contributed to $42.62 \%$ of the local P. vivax malaria cases reported in the country (Table 2).

The distribution of $P$. falciparum malaria also showed spatial clustering during 2005-2014. From 2005 to 2011, the most likely cluster was consistently detected in western Yunnan province along the China-Myanmar border (Fig. 3). The number of counties per most likely clusters during this period ranged from 7 to 13 . The cluster with the highest number of counties $(n=13)$ was detected in 2006. This cluster contributed to more than half of the local $P$. falciparum malaria cases reported each year from 2005 to 2011 (i.e., 78.52, 77.54, 65.97, 58.22, 55.00, 54.61 and $46.71 \%$, respectively). During 2012-2014, the most likely spatial cluster was detected in the counties of central Guangxi (in 2012 \& 2013) and Anhui (in 2014). The most likely spatial cluster detected during this period 

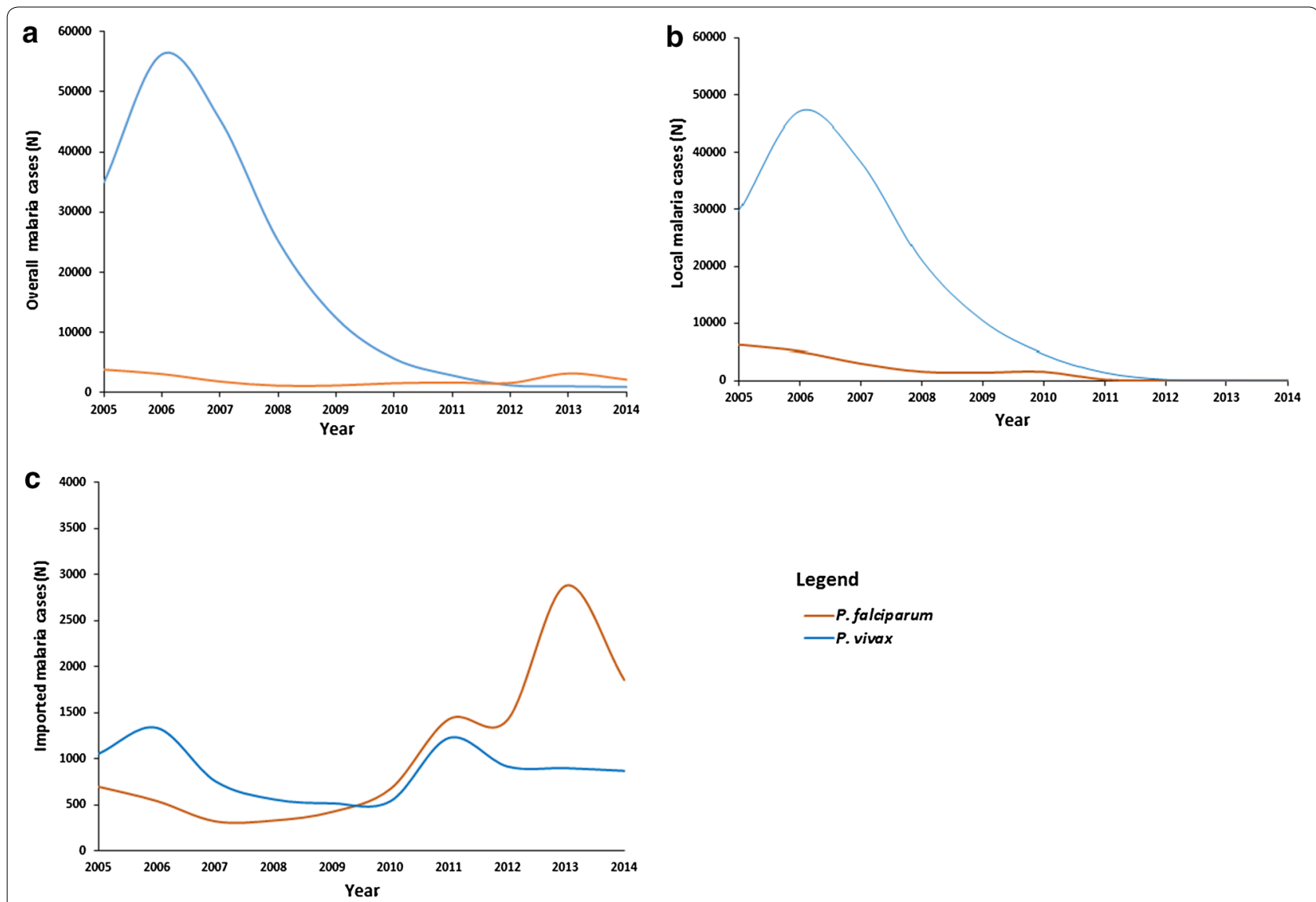

Fig. 1 Annual trend of overall, local and imported P. vivax and $P$. falciparum malaria in China from 2005 to 2014. a Overall trend of $P$. vivax and $P$. falciparum malaria. b Annual trends of local P. vivax and P. falciparum malaria. c Annual trends of imported P. vivax and P. falciparum malaria

Table 1 Spatial autocorrelation (Global Moran's $I$ ) of $P$. vivax and $P$. falciparum in China during 2005-2014

\begin{tabular}{|c|c|c|c|c|c|c|}
\hline \multirow[t]{2}{*}{ Year } & \multicolumn{3}{|c|}{ P. vivax } & \multicolumn{3}{|c|}{ P. falciparum } \\
\hline & $I$ & Z & $p$ value & $I$ & Z & $p$ value \\
\hline 2005 & 0.42 & 20.1 & $<0.001$ & 0.11 & 43.01 & $<0.001$ \\
\hline 2006 & 0.53 & 21.5 & $<0.001$ & 0.10 & 52.19 & $<0.001$ \\
\hline 2007 & 0.49 & 21.25 & $<0.001$ & 0.07 & 42.35 & $<0.001$ \\
\hline 2008 & 0.53 & 29.24 & $<0.001$ & 0.08 & 51.45 & $<0.001$ \\
\hline 2009 & 0.52 & 24.03 & $<0.001$ & 0.08 & 45.56 & $<0.001$ \\
\hline 2010 & 0.34 & 20.85 & $<0.001$ & 0.07 & 38.32 & $<0.001$ \\
\hline 2011 & 0.28 & 17.97 & $<0.001$ & 0.05 & 24.18 & $<0.001$ \\
\hline 2012 & 0.19 & 14.86 & $<0.001$ & 0.02 & 9.42 & $<0.001$ \\
\hline 2013 & 0.09 & 5.90 & $<0.001$ & 0.01 & 1.86 & $<0.01$ \\
\hline 2014 & 0.07 & 38.22 & $<0.001$ & 0.03 & 16.61 & $<0.001$ \\
\hline
\end{tabular}

I Moran's Index, Z z-score

included a small number of counties. However, 48.90\% of the total counties per secondary spatial clusters were detected during 2012-2014. Overall, the spatial cluster areas contributed to more than half (53.2\%) of the local P. falciparum malaria cases reported during 2012-2014 (Table 3). 


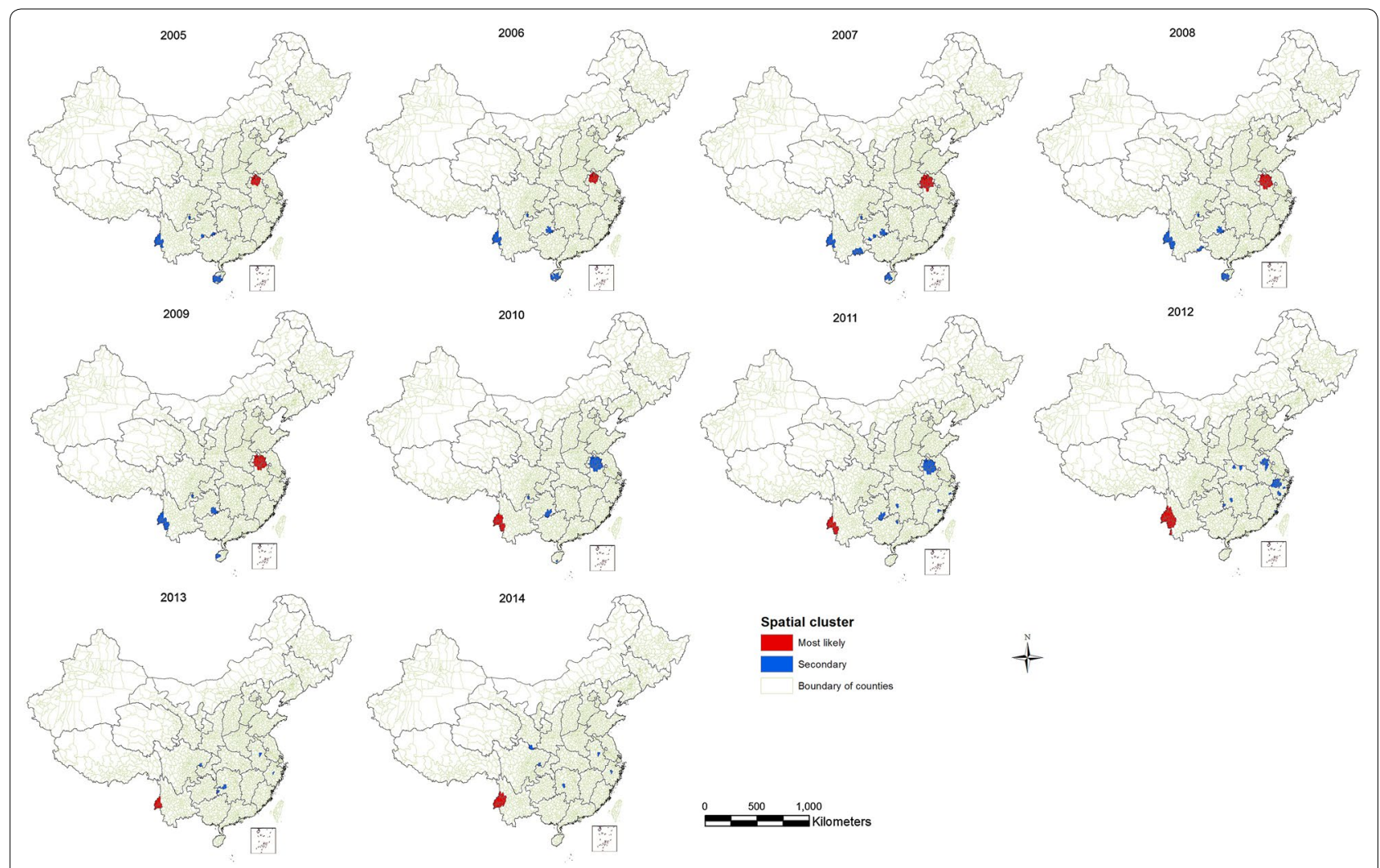

Fig. 2 The locations of the detected spatial clusters of P. vivax malaria in China from 2005 to 2014

\section{Space-time clustering}

The space-time scan statistics identified both the most likely and secondary clusters of locations with an elevated risk of $P$. vivax and $P$. falciparum malaria (Table 4 ). The most likely space-time cluster of $P$. vivax encompassing 32 counties was in a northern part of Anhui province in 2005-2009 (Fig. 4a). A secondary space-time cluster of $P$. vivax encompassing 20 counties was identified in the western part of Yunnan (2005-2009) and Hainan (2005-2008) provinces. For P. falciparum, the most likely space-time cluster included 10 counties in the western part of Yunnan province during the same period. A secondary space-time cluster of $P$. falciparum was detected in Anhui, Guangxi, and Zhejiang province from 2012 to 2013 (Fig. 4b).

\section{Discussion}

Using a surveillance dataset of 10 years, the present study demonstrated substantial changes occurring with respect to annual trends and the geographical distribution of malaria in China. The annual number of $P$. vivax malaria cases showed a considerable decrease, especially after 2006 when the NMCP was launched [12]. However, the number of $P$. falciparum cases remained relatively stable at a higher level. $P$. falciparum cases malaria peaked in 2005, fell until 2008, then slightly increased, and has dominated cases since 2012. The steady decline in $P$. vivax cases and increase in $P$. falciparum cases was similar to that noted previously [5].

The present study also indicated a shrinking in the geographical distribution of $P$. vivax malaria and a substantial expansion in areas with $P$. falciparum. These findings coincide with previous research [15, 35]. Different interventions have been formulated with the aim of controlling malaria in China. These have been effectively implemented with support from the Global Fund to Fight AIDS, Tuberculosis and Malaria (GFATM) [36]. This may have increased the ability of counties to effectively control local malaria transmission dominated by $P$. vivax $[15$, 16]. A possible reason for the geographical expansion of $P$. falciparum malaria could be the rising number of overseas imported malaria cases in recent years [14,37]. As of 2013, $97.9 \%$ of the national malaria cases reported came from overseas [15]. Most overseas-imported malaria in recent years was $P$. falciparum. One study conducted in Jiangsu province showed that $P$. falciparum accounted for $79.8 \%$ of the total malaria cases imported to the province during 2001-2011 [38]. In recent years, a dramatic 
Table 2 Pure spatial clustering of $P$. vivax cases in China during 2005-2014

\begin{tabular}{|c|c|c|c|c|c|c|c|c|}
\hline Year & Clusters & Latitude & Longitude & $\mathbf{N}$ & Observed cases & Expected cases & RR & p value \\
\hline \multirow[t]{2}{*}{2005} & Most likely & 33.68399 & 116.72312 & 15 & 15,241 & 570.93 & 48.44 & 0.001 \\
\hline & Secondary & 24.03683 & 97.80955 & 22 & 5590 & 105.74 & 63.35 & 0.001 \\
\hline \multirow[t]{2}{*}{2006} & Most likely & 33.68399 & 116.72312 & 15 & 35,053 & 958.00 & 97.94 & 0.001 \\
\hline & Secondary & 24.03683 & 97.80955 & 23 & 5595 & 177.28 & 34.99 & 0.001 \\
\hline \multirow[t]{2}{*}{2007} & Most likely & 33.21876 & 116.58207 & 35 & 31,436 & 1543.33 & 66.53 & 0.001 \\
\hline & Secondary & 24.03683 & 97.80955 & 29 & 3122 & 143.51 & 23.32 & 0.001 \\
\hline \multirow[t]{2}{*}{2008} & Most likely & 33.03381 & 117.03400 & 32 & 15,766 & 717.31 & 57.44 & 0.001 \\
\hline & Secondary & 24.03683 & 97.80955 & 28 & 2091 & 109.56 & 20.73 & 0.001 \\
\hline \multirow[t]{2}{*}{2009} & Most likely & 33.03381 & 117.03400 & 32 & 6533 & 345.07 & 39.07 & 0.001 \\
\hline & Secondary & 24.03683 & 97.80955 & 21 & 1561 & 53.42 & 33.31 & 0.001 \\
\hline \multirow[t]{2}{*}{2010} & Most likely & 24.03683 & 97.80955 & 13 & 1320 & 30.02 & 57.20 & 0.001 \\
\hline & Secondary & 33.03381 & 117.03400 & 39 & 1872 & 151.63 & 18.03 & 0.001 \\
\hline \multirow[t]{2}{*}{2011} & Most likely & 24.03683 & 97.80955 & 12 & 799 & 11.67 & 95.57 & 0.001 \\
\hline & Secondary & 33.03381 & 117.03400 & 39 & 678 & 72.60 & 12.01 & 0.001 \\
\hline \multirow[t]{2}{*}{2012} & Most likely & 24.03683 & 97.80955 & 23 & 516 & 9.26 & 101.22 & 0.001 \\
\hline & Secondary & 28.20380 & 120.13838 & 22 & 20 & 1.06 & 19.19 & 0.001 \\
\hline \multirow[t]{2}{*}{2013} & Most likely & 24.85646 & 97.91907 & 7 & 289 & 2.31 & 183.35 & 0.001 \\
\hline & Secondary & 32.00298 & 117.56783 & 9 & 22 & 0.76 & 29.67 & 0.001 \\
\hline \multirow[t]{2}{*}{2014} & Most likely & 25.27506 & 98.49739 & 14 & 324 & 4.49 & 113.70 & 0.001 \\
\hline & Secondary & 32.94082 & 104.77725 & 7 & 47 & 0.19 & 261.31 & 0.001 \\
\hline
\end{tabular}

$N$ number of counties per cluster, $R R$ indicates relative risk for malaria case in the location

increase in overseas investments increased the number of Chinese persons working abroad and paved the way for international travel to and from countries where $P$. falciparum is highly endemic. In 2012, about 0.5 million people left the country for work and 83.2 million for other reasons. Compared to 2010, an increase of 24.6 and $44.9 \%$ were observed, respectively [39]. Available evidence indicated that $P$. falciparum was mostly imported via Chinese people returning from Africa [17, 38, 40-44].

Identifying a high-risk area is crucial for spatial targeting of interventions against malaria transmission. Spatial cluster analysis identified most likely clusters of $P$. vivax malaria located in North Anhui province from 2005 to 2009. The area where most likely clusters of $P$. vivax were identified in this study was similar to the previous transmission foci detected in the northern part of Anhui province [18], where An. sinensis is a principal vector. A study suggested that increased vector capacity of $A n$. sinensis related with the mosquito host reduction (livestock), and human behavioural change contributed to $P$. vivax malaria outbreak in Huaiyuan county of Anhui province [45]. This area, especially north of the Huai River, is one of the high-risk areas with unstable malaria transmission [46], possibly due to environmental conditions associated with geographical location $[11,14,18]$. Malaria transmission in this province has been an important issue in China, responsible for the outbreak in 2006 which was dominated by P. vivax [11]. From 2006 to 2009, Anhui had been the number one province in China in terms of number of malaria cases [11, 13, 47].

The present study also identified most likely spatial clusters of $P$. vivax malaria in western Yunnan province, along the China-Myanmar border after 2009. The cluster persisted for five years (2010-2014), contributing more than half the total number of $P$. vivax cases reported each year. Although the high-risk area identified in this area agrees with previous studies [20, 48, 49], a shift of the geographical location from Anhui to Yunnan province after 2009 is new. The reduction in Anhui may be explained by intensive malaria control in the central China provinces [50]. Since the initiation of the NMEP in $2010[12,51]$, national malaria, especially local cases greatly declined $[8,15]$. One study showed that overall malaria cases in Anhui province decreased by 65.5\% (in 2011) compared to those in 2010 [9]. The total number of $P$. vivax cases in the country, therefore, decreased by $57.7 \%$ in one year, with most of the local cases in Yunnan province [16]. The province remained endemic, ranking first in the country in terms of an overall number of malaria cases $[8,9,15,52]$, particularly $P$. vivax [16]. For example, $73.1 \%(171 / 234)$ of the national $P$. vivax malaria cases in 2012 were contributed by Yunnan [16], and $P$. vivax malaria appeared to be dominant along the China-Myanmar border [53]. Malaria transmission in 


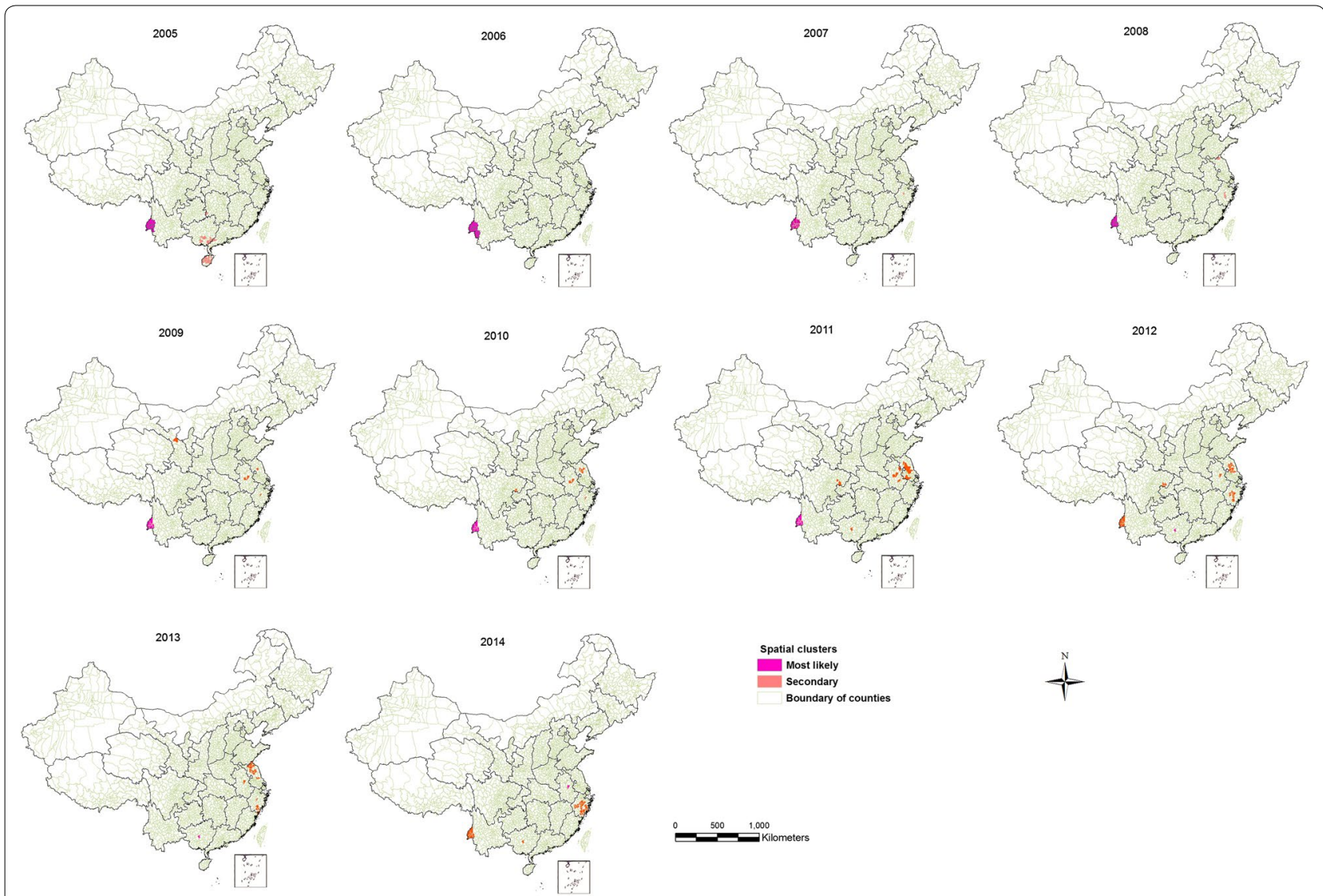

Fig. 3 Location of the detected spatial clusters of P. falciparum malaria cases in China from 2005 to 2014

Table 3 Pure spatial clustering of $P$. falciparum cases in China during 2005-2014

\begin{tabular}{|c|c|c|c|c|c|c|c|c|}
\hline Year & Clusters & Latitude & Longitude & $N$ & Observed cases & Expected cases & RR & $\overline{p \text { value }}$ \\
\hline \multirow[t]{2}{*}{2005} & Most likely & 24.73918 & 98.31948 & 10 & 2545 & 27.18 & 335.72 & 0.001 \\
\hline & Secondary & 21.08838 & 110.32950 & 25 & 198 & 103.24 & 1.97 & 0.001 \\
\hline 2006 & Most likely & 24.03683 & 97.80955 & 13 & 2265 & 28.68 & 348.14 & 0.001 \\
\hline \multirow[t]{2}{*}{2007} & Secondary & 24.73918 & 98.31948 & 9 & 1118 & 12.66 & 249.20 & 0.001 \\
\hline & Most likely & 29.52352 & 119.90339 & 1 & 20 & 1.55 & 13.08 & 0.001 \\
\hline \multirow[t]{2}{*}{2008} & Secondary & 24.85646 & 97.91907 & 7 & 590 & 5.02 & 255.22 & 0.001 \\
\hline & Most likely & 34.55674 & 118.78395 & 4 & 26 & 2.85 & 9.33 & 0.001 \\
\hline \multirow[t]{2}{*}{2009} & Secondary & 24.85646 & 97.91907 & 7 & 508 & 4.97 & 192.20 & 0.001 \\
\hline & Most likely & 37.52641 & 103.33228 & 8 & 22 & 0.92 & 24.47 & 0.001 \\
\hline \multirow[t]{2}{*}{2010} & Secondary & 24.85646 & 97.91907 & 7 & 529 & 6.35 & 133.89 & 0.001 \\
\hline & Most likely & 30.53620 & 106.41193 & 11 & 61 & 4.63 & 13.74 & 0.001 \\
\hline \multirow[t]{2}{*}{2011} & Secondary & 24.85646 & 97.91907 & 7 & 213 & 5.67 & 45.08 & 0.001 \\
\hline & Most likely & 32.10585 & 118.76075 & 38 & 297 & 54.25 & 6.88 & 0.001 \\
\hline \multirow[t]{2}{*}{2012} & Secondary & 23.52086 & 108.63520 & 1 & 112 & 0.75 & 167.01 & 0.001 \\
\hline & Most likely & 24.85646 & 97.91907 & 31 & 144 & 4.39 & 37.48 & 0.001 \\
\hline \multirow[t]{2}{*}{2013} & Secondary & 23.52086 & 108.63520 & 1 & 955 & 1.46 & 1081.9 & 0.001 \\
\hline & Most likely & 32.00298 & 117.56783 & 21 & 134 & 3.58 & 39.53 & 0.001 \\
\hline \multirow[t]{2}{*}{2014} & Secondary & 32.00298 & 117.56783 & 1 & 90 & 1.82 & 52.79 & 0.001 \\
\hline & Most likely & 23.52086 & 108.63520 & 32 & 56 & 0.75 & 77.63 & 0.001 \\
\hline
\end{tabular}

$N$ number of counties per cluster, $R R$ indicates relative risk for malaria case in the location 
Table 4 Space-time clustering of $P$. vivax and $P$. falciparum malaria in China, 2005-2014

\begin{tabular}{lllllllrrr}
\hline Malaria parasites & Clusters & Latitude & Longitude & Start/end date & N & Observed cases & Exp. case & RR & p value \\
\hline P. vivax & Most likely & 24.73918 & 98.31948 & $2005 / 2009$ & 10 & 7011 & 67.29 & 170.70 & 0.001 \\
& Secondary & 23.52086 & 108.63520 & $2013 / 2013$ & 24 & 955 & 1.13 & 895.75 & 0.001 \\
P. falciparum & Most likely & 33.03381 & 117.03400 & $2005 / 2009$ & 32 & 10,829 & 2538.07 & 103.88 & 0.001 \\
& Secondary & 24.03683 & 97.80955 & $2005 / 2009$ & 26 & 18,919 & 385.80 & 54.61 & 0.001 \\
\hline
\end{tabular}

$N$ number of counties per cluster, $R R$ indicates relative risk for malaria case in the location. Obs. case observed number of case in the cluster, Exp. case expected number of cases in the cluster

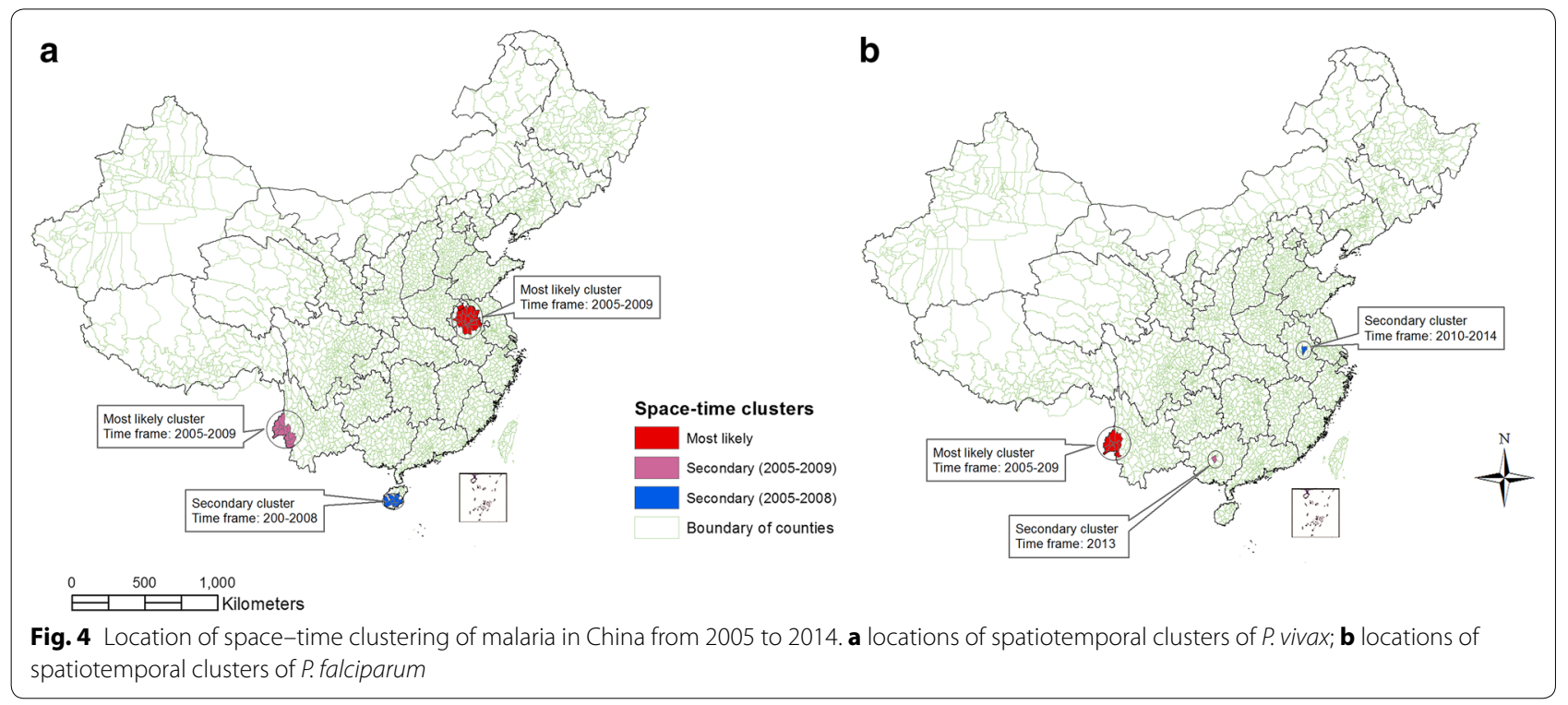

this area is a major concern in the disease elimination stage. This could be attributed to environmental conditions conducive for transmission and efficiency of the dominant $A n$. minimus vectors in this area [17]. Unlike An. sinensis [54, 55], An. minimus shows a strong attraction to human than other hosts [56] and has a high human blood index [57] which has an implication for vector control interventions even though An. minimus is endophilic, endophagic, and susceptible to insecticides $[55,58]$. Human behavioural factors [59] could be another reason for malaria transmission and control in this area. For example, some counties of Yunnan province along the international border have been recognized as high-risk areas in China because of sharing a boundary with malaria endemic countries which put them at risk of reintroduction $[20,60]$. One study revealed that imported P. vivax to China had increased between 2004 and 2012, most of which were from malaria endemic countries of South East Asia (Myanmar, Cambodia and Laos) [16]. $59.3 \%$ of the total $P$. vivax malaria cases imported from South East Asia $(n=697)$ were introduced from Myanmar, and $56.7 \%(\mathrm{n}=6832)$ of the total introduced $P$. vivax cases $(\mathrm{n}=12,060)$ were in Yunnan [17]. However, other evidence shows lack of significant association between travel to Myanmar and transmission of $P$. vivax along the China-Myanmar border [53]. Residents in this area have a relatively low educational level, limited knowledge of malaria transmission and utilization of personal protection, especially during outdoor activities [61, 62], and exhibit poor treatment-seeking behaviour [62]. These factors could contribute to sustained malaria transmission in this area [17].

Similarly, the current study identified the most likely spatial clusters of $P$. falciparum malaria in the western Yunnan province along the China-Myanmar border every year from 2005 to 2011 . This is consistent with previous studies $[20,48,49]$. This is the only area where the local $P$. falciparum cases were reported by several studies $[9,15]$. It implies that these counties are at high-risk of achieving stable falciparum malaria transmission. Most of the national P. falciparum cases during 2005-2011 were reported from Yunnan province, although counties were not frequently specified [9, 11, 47, 52]. In addition to behavioural and lifestyle factors for malaria transmission [61], travel to Myanmar was significantly associated with acquiring $P$. falciparum infection, indicating 
cross-border movement is a key factor for the stable transmission of $P$. falciparum in the China-Myanmar border $[17,53,61,62]$. However, $84.5 \%$ of the imported $P$. falciparum cases were imported from Africa. Climatic factors have also been found to be an important factor for malaria transmission in this area [48]. According to one study [20], temperature was significantly associated with vivax malaria in clustered areas of Yunnan province. Further research is required to better understand the importance of climatic factors in the spatial distribution of malaria in China.

The present study also identified high-risk areas for $P$. falciparum in central Anhui province from 2013 to 2014. This foci (Feidong county) is different from the previously identified high-risk area in the Northern Anhui province [18], indicating spatial variation in foci of $P$. falciparum cases, was and its less importance in this area than $P$. vivax [11]. In the present study, the most likely spatial cluster of $P$. falciparum was not detected in Anhui province until 2012. However, a spatial cluster of $P$. vivax was observed consistently in the northern Anhui province from 2005 to 2009 . This result verified that P. falciparum played a relatively insignificant role in the previously identified high-risk area in Anhui province [18].

The high-risk area for $P$. falciparum malaria shifted from Yunnan to Anhui province, and very large secondary clusters were detected in some counties of the northern and eastern provinces, especially after 2011. This could be attributed to the increased proportion of overseas imported malaria from the parasite endemic countries in recent years $[14,46]$. P. falciparum dominates overseas imported malaria cases, which are distributed in different parts of China, including non-endemic provinces. Nevertheless, the study of disease clustering focused only on local malaria cases. Spatial variations among $P$. vivax and P. falciparum malaria followed different patterns indicating differences in the biological features of parasites, which might have facilitated their transmission. Climatic factors are associated with an increased risk of malaria because of their impact on vector activities and the parasite incubation period [63]. Compared to P. vivax, P. falciparum requires a slightly higher temperature for parasite development. The minimum threshold temperature for $P$. falciparum and $P$. vivax are approximately 18 and $15{ }^{\circ} \mathrm{C}$, respectively [64], indicating increased opportunity for $P$. falciparum to spread to previously cooler areas, following global climate change. Further studies are required to fully understand the risk factors driving spatial shifting and geographic expansion of $P$. falciparum across mainland China.

The most likely space-time cluster of $P$. vivax malaria was detected in the northern Anhui province. These areas coincided with results of the purely spatial analysis in this study as well those of previous studies [18]. The time frame for all significant space-time clusters of $P$. vivax malaria was 2005-2009. This implies a declining burden of $P$. vivax malaria in China since 2010. Control interventions, especially those following the establishment of the Chinese NMEP $[12,51]$ are a likely factor in this substantial reduction of this malaria.

For P. falciparum, the most likely space-time cluster was detected in western Yunnan province during 20052009. Although this is consistent with the previous study [65], the secondary space-time cluster was scattered mostly in the eastern and north-eastern provinces after 2012 (Fig. 4b). This could imply the spreading of $P$. falciparum malaria to previously non-endemic areas, probably due to an increased number of overseas-imported P. falciparum $[8,15,35]$. Although an imported case can be distributed randomly to any provinces, the present study showed a greater expansion of $P$. falciparum to the east and northeast of China than to any other parts of the country. A better understanding of the direction of malaria expansion or spatial change and underlying risk factors for the malaria transmission in these formerly non-endemic areas is important for the malaria elimination goal of China.

This study is the first to identify the spatial and spatiotemporal distribution of $P$. vivax and P. falciparum at the national level in China. A separate analysis was conducted for both important malaria parasites in the country. The study identified high-risk areas and the spatial extent of both $P$. vivax and $P$. falciparum. Spatial and space-time scan statistics were performed using SaTScan software [66]. These techniques were designed particularly to perform spatial clustering of disease or health related events, and to test the statistical significance of clustering under the null hypothesis of a random distribution of the diseases in space, time and space-time [66, 67]. These techniques are most effective at identifying disease clusters [68], and have been widely used in fields of epidemiology for similar purpose [18, 19, 21, 24, 25].

Although purely spatial scan statistics are most effective at identifying a cluster of malaria with a circular shape [68], the spatial distribution of the disease may not always assume this shape and some irregularly shaped clusters might have been undetected.

\section{Conclusion}

Overall, the spatial and space-time cluster detection statistics identified a high-risk areas for both $P$. vivax and $P$. falciparum malaria. The counties in the clusters should be given priority in the control programs, and for further operational research. Both malaria types showed significant spatial and spatiotemporal variations. Contrary to $P$. vivax, the high-risk areas for $P$. falciparum malaria 
shifted from southwest to east of China. Thus, further studies are required to examine spatial changes in the risk of malaria transmission and identify the underlying causes of elevated risk in emerging high-risk areas.

\section{Additional files}

Additional file 1. Distribution maps of imported malaria cases.

Additional file 2. Number of malaria cases imported from other countries, 2005-2014

\section{Abbreviations}

CISDCP: China information system for disease control and prevention; NMCP: National Malaria Control Programme; NMEP: National Malaria Elimination Programme; CDC: Center for Disease Control and Prevention; I: Moran's index; Z: z-score; RR: indicates relative risk; AIDS: acquired human immuno deficiency syndrome.
\end{abstract}

\section{Authors' contributions}

SH and YG designed the study protocol, WZ and JG provided original dataset, SL and LC compiled and prepared the dataset for analysis. SH and YG did the analysis and interpretation of the results, and drafted the manuscript. YG, GW, $\mathrm{SL}$, and WZ critically revised the manuscript for intellectual content. All authors read and approved the final manuscript.

\section{Acknowledgements}

$\mathrm{SH}$ is a Ph.D student supported by an International Postgraduate Research (IPRS) and The University of Queensland Scholarship; YG is supported by the Career Development Fellowship of Australian National Health and Medical Research Council (\#APP1107107)

\section{Competing interests}

The authors declare that they have no competing interests.

\section{Availability of data and materials}

The dataset of this article is available upon request.

\section{Ethical approval}

Prior to the start of this research, the study protocol was approved by the Ethics Committee of the University of Queensland, Australia. The data was de-identified and aggregated to county-level prior to commencement of the data analysis.

Received: 1 September 2016 Accepted: 5 December 2016 Published online: 19 December 2016

\section{References}

1. Cox FE. Modern parasitology. A textbook of parasitology. Oxford: Blackwell Scientific Publications; 1982.

2. WHO. World Malaria Report. Geneva: World Health Organization; 2015 http://apps.who.int/iris/bitstream/10665/200018/1/9789241565158_eng. pdf?ua=1. Accessed 20 Jan 2015.

3. Hommel M. Towards a research agenda for global malaria elimination. Malar J. 2008;7(1):S1.

4. WHO. Global Health Observatory $(\mathrm{GHO})$ data. http://www.who.int/gho/ malaria/en/. Accessed 2 Oct 2015.

5. Zhang Q, Lai S, Zheng C, Zhang H, Zhou S, Hu W, et al. The epidemiology of Plasmodium vivax and Plasmodium falciparum malaria in China, 2004-2012: from intensified control to elimination. Malar J. 2014;13:419.

6. Huang JX, Xia ZG, Zhou SS, Pu XJ, Hu MG, Huang DC, et al. Spatio-temporal analysis of malaria vectors in national malaria surveillance sites in China. Parasit Vectors. 2015:8:146.
7. Yin JH, Zhou SS, Xia ZG, Wang RB, Qian YJ, Yang WZ, et al. Historical patterns of malaria transmission in China. Adv Parasitol. 2014;86:1-19.

8. Xia ZG, Feng J, Zhou SS. Malaria situation in the People's Republic of China in 2012. Chin J Parasitol Parasitic Diseases. 2013;31:413-8 (in Chinese).

9. Xia ZG, Yang MN, Zhou SS. Malaria situation in the People's Republic of China in 2011. Chin J Parasitol Parasit Dis. 2012;30:419-22 (in Chinese)

10. Lu G, Zhou S, Horstick O, Wang X, Liu Y, Muller O. Malaria outbreaks in China (1990-2013): a systematic review. Malar J. 2014;13:269.

11. Zhou SS, Wang Y, Tang LH. Malaria situation in the People's Republic of China in 2006. Chin J Parasitol Parasit Dis. 2007;25:439-41 (in Chinese).

12. China Ministry of Health. From malaria control to elimination: a revised national malaria strategy 2010-2015. China Ministry of Health: Beijing; 2012.

13. Zhou SS, Wang Y, Xia ZG. Malaria situation in the People's Republic Of China in 2009. Chin J Parasitol Parasit Dis. 2011;29:1-3 (in Chinese)

14. Feng J, Xiao H, Xia Z, Zhang L, Xiao N. Analysis of malaria epidemiological characteristics in the People's Republic of China, 2004-2013. Am J Trop Med Hyg. 2015;93:293-9.

15. Zhang L, Feng J, Xia ZG. Malaria situation in the People's Republic of China in 2013. Chin J Parasitol Parasit Dis. 2014;32:407-13 (in Chinese).

16. Feng J, Xiao H, Zhang L, Yan H, Feng X, Fang W, et al. The Plasmodium vivax in China: decreased in local cases but increased imported cases from Southeast Asia and Africa. Sci Rep. 2015;5:8847.

17. Xia ZG, Zhang L, Feng J, Li M, Feng XY, Tang LH, et al. Lessons from malaria control to elimination: case study in Hainan and Yunnan provinces. Adv Parasitol. 2014;86:47-79.

18. Zhang W, Wang L, Fang L, Ma J, Xu Y, Jiang J, et al. Spatial analysis of malaria in Anhui province China. Malar J. 2008;7:206.

19. Xia J, Cai S, Zhang H, Lin W, Fan Y, Qiu J, et al. Spatial, temporal, and spatiotemporal analysis of malaria in Hubei Province, China from 2004-2011. Malar J. 2015;14:145.

20. Hui F-M, Xu B, Chen Z-W, Cheng X, Liang L, Huang H-B, et al. Spatio-temporal distribution of malaria in Yunnan Province China. Am J Trop Med Hyg. 2009;81:503-9.

21. Wen L, Li C, Lin M, Yuan Z, Huo D, Li S, et al. Spatio-temporal analysis of malaria incidence at the village level in a malaria-endemic area in Hainan China. Malar J. 2011:10:88.

22. Wen L, Shi RH, Fang LQ, Xu DZ, Li CY, Wang Y, et al. Spatial epidemiological study on malaria epidemics in Hainan province. Chin J Parasitol Parasit Dis. 2008;29:581-5 (in Chinese)

23. Caprarelli G, Fletcher S. A brief review of spatial analysis concepts and tools used for mapping, containment and risk modelling of infectious diseases and other illnesses. Parasitology. 2014;141:581-601.

24. Xiao D, Long Y, Wang S, Fang L, Xu D, Wang G, et al. Spatiotemporal distribution of malaria and the association between its epidemic and climate factors in Hainan, China. Malar J. 2010;9:185.

25. Zhang Y, Liu Q, Rs L, Xb L, Zhou G, Jiang J, et al. Spatial-temporal analysis of malaria and the effect of environmental factors on its incidence in Yongcheng, China, 2006-2010. BMC Public Health. 2012;12:544.

26. Population reference bureau. Population of provinces, municipalities, and autonomous regions, 2010 census of China, and Change in the percent distribution by area, 2000-2010. 2011. http://www.prb.org/pdf11/china2010-census-results-table.pdf. Accessed 25 Dec 2015.

27. Wang L, Wang Y, Yang G, Ma J, Wang L, Qi X-P. China information system for disease control and prevention (CISDCP). 2013.

28. Feng $X Y$, Xia ZG, Vong S, Yang WZ, Zhou SS. Surveillance and response to drive the national malaria elimination program. Adv Parasitol. 2014;86:81-108.

29. R Core Team. R. A language and environment for statistical computing. Vienna, Austria: R Foundation for Statistical Computing; 2015. http:// www.R-project.org/. Accessed 2 Nov 2015.

30. ESRI. ArcGIS Desktop: Release 10.3.1. Redlands: Environmental Systems Research Institute; 2014.

31. Rao HX, Zhang X, Zhao L, Yu J, Ren W, Zhang XL, et al. Spatial transmission and meteorological determinants of tuberculosis incidence in Qinghai Province, China: a spatial clustering panel analysis. Infect Dis Poverty. 2016;5:45.

32. Wu W, Guo J, Guan P, Sun Y, Zhou B. Clusters of spatial, temporal, and space-time distribution of hemorrhagic fever with renal syndrome in Liaoning Province Northeastern China. BMC Infect Dis. 2011;11:229. 
33. Kulldorff M, Rand K, Gherman G, Williams G, DeFrancesco D. SaTScan $\checkmark$ 2.1: software for the spatial and space-time scan statistics. Bethesda: National Cancer Institute; 1998.

34. Kulldorff M. SaTScanTM user guide for version 8. 0. 2009.

35. Yin JH, Yang MN, Zhou SS, Wang Y, Feng J, Xia ZG. Changing malaria transmission and implications in China towards National Malaria Elimination Programme between 2010 and 2012. PLoS ONE. 2013;8:e74228.

36. Wang RB, Zhang QF, Zheng B, Xia ZG, Zhou SS, Tang LH, et al. Transition from control to elimination: impact of the 10-year global fund project on malaria control and elimination in China. Adv Parasitol. 2014;86:289-318.

37. Xia J, Cai SX, Lin W, Sun LC, Li KJ, Pei SJ, et al. Epidemiological analysis of malaria prevalence in Hubei Province in 2013. Chin J Schistosomiasis Control. 2015;27:186-8 (in Chinese).

38. Liu Y, Hsiang MS, Zhou H, Wang W, Cao Y, Gosling RD, et al. Malaria in overseas labourers returning to China: an analysis of imported malaria in Jiangsu Province, 2001-2011. Malar J. 2014;13:29.

39. Feng J, Yan H, Feng $X$, Zhang L, Li M, Xia Z, et al. Imported malaria in China, 2012. Emerg Infect Dis. 2014;20:1778-80.

40. Chen H, Yao L, Zhang L, Zhang X, Lu Q, Yu K, et al. Malaria in Zhejiang Province, China, from 2005 to 2014. Am J Trop Med Hyg. 2015;93:305-9.

41. Liu YB, Cao J, Zhou HY, Wang WM, Cao YY, Gao Q. Analysis of overseas imported malaria situation and implication for control in Jiangsu Province, PR China. Chin J Schistosomiasis Control. 2013;25:44-7 (in Chinese).

42. Pan B, Ruan CW, Lin RX, Pei FQ, Zhang QM. Epidemiological analysis of malaria epidemic situation in Guangdong Province, 2011. Chin J Schistosomiasis Control. 2013;25:40-3 (in Chinese).

43. Yang CY, Lu DL, Zhou RM, Liu Y, Zhang HW, Zhao YL. Malaria situation in Henan Province in 2013. Chin J Parasitol Parasit Dis. 2014;32:370-2 (in Chinese).

44. Zhang HW, Su YP, Zhao XD, Yan QY, Liu Y, Chen JS. Imported falciparum malaria situation in Henan Province during 2005-2009. Chin J Parasitol Parasit Dis. 2010;28:476-7 (in Chinese).

45. Pan JY, Zhou SS, Zheng X, Huang F, Wang DQ, Shen YZ, et al. Vector capacity of Anopheles sinensis in malaria outbreak areas of central China. Parasit Vectors. 2012;5:136.

46. Xu X, Li WD, Jiang JJ, Zhang T, Wang JJ. Analysis of malaria epidemic characteristics in Anhui Province during 1999-2013. Chin J Parasitol Parasit Dis. 2015;33:1-6 (in Chinese).

47. Zhou SS, Wang Y, Fang W, Tang LH. Malaria situation in the People's Republic Of China in 2007. Chin J Parasitol Parasit Dis. 2008;26:401-3 (in Chinese).

48. Bi Y, Yu W, Hu W, Lin H, Guo Y, Zhou XN, et al. Impact of climate variability on Plasmodium vivax and Plasmodium falciparum malaria in Yunnan Province China. Parasit Vectors. 2013;6:357.

49. Clements AC, Barnett AG, Cheng ZW, Snow RW, Zhou HN. Spacetime variation of malaria incidence in Yunnan province China. Malar J. 2009:8:180.

50. Liu Y, Su Y-p, Zhang H-w. Analysis of malaria situation in five provinces of Jiangsu, Shandong, Henan, Anhui and Hubei in 2006 China. Trop Med. 2008;8:198-208 (in Chinse).
51. China Ministry of Health. Action plan of China malaria elimination (2010-2020). Ministry of Health: Beijing; 2010.

52. Zhou SS, Wang Y, Li Y. Malaria situation in the People's Republic of China in 2010. Chin J Parasitol Parasit Dis. 2011;29:401-3 (in Chinese).

53. Zhou G, Sun L, Xia R, Duan Y, Xu J, Yang H, et al. Clinical malaria along the China-Myanmar border, Yunnan Province, China, January 2011-August 2012. Emerg Infect Dis. 2014;20:675-8.

54. Liu XB, Liu QY, Guo YH, Jiang JY, Ren DS, Zhou GC, et al. The abundance and host-seeking behavior of culicine species (Diptera: Culicidae) and Anopheles sinensis in Yongcheng city People's Republic of China. Parasit Vectors. 2011:4:221.

55. Chow C-Y. Malaria vectors in China. Chin J Entomol. 1991;6:67-79.

56. Tisgratog R, Tananchai C, Juntarajumnong W, Tuntakom S, Bangs MJ, Corbel V, et al. Host feeding patterns and preference of Anopheles minimus (Diptera: Culicidae) in a malaria endemic area of western Thailand: baseline site description. Parasit Vectors. 2012;5:114.

57. Yu G, Yan G, Zhang N, Zhong D, Wang Y, He Z, et al. The Anopheles community and the role of Anopheles minimus on malaria transmission on the China-Myanmar border. Parasit Vectors. 2013;6:264.

58. Cui F, Raymond M, Qiao CL. Insecticide resistance in vector mosquitoes in China. Pest Manag Sci. 2006;62:1013-22.

59. Spielman A. The behavioural and social aspects of malaria and its control. UNDP/World Bank/WHO. Special programme for research \& training in tropical diseases (TDR). 2003.

60. Feng J, Xia ZG, Vong S, Yang WZ, Zhou SS, Xiao N. Preparedness for malaria resurgence in China: case study on imported cases in 2000-2012. Adv Parasitol. 2014;86:231-65.

61. Ying D, Xing-rong W, Yu-bin Z, Chun-lin L, Xian-hua W, Zheng-you W, et al. Study on malaria knowledge in border population, Yunnan Province. Chin J Schistosomiasis Control. 2007;1:20 (in Chinese).

62. Moore SJ, Min X, Hill N, Jones C, Zaixing Z, Cameron MM. Border malaria in China: knowledge and use of personal protection by minority populations and implications for malaria control: a questionnaire-based survey. BMC Public Health. 2008;8:344.

63. Wang D, Li S, Cheng Z, Xiao N, Cotter C, Hwang J, et al. Transmission risk fromimported Plasmodium vivax malaria in the China-Myanmar border region. Emerg Infect Dis. 2015;21:1861.

64. Patz JA, Olson SH. Malaria risk and temperature: influences from global climate change and local land use practices. Proc Natl Acad Sci USA. 2006;103:5635-6.

65. An G. Influence of climate on malaria in China. Penn McNair Res J. 2011;3(1):1.

66. Lin H, Lu L, Tian L, Zhou S, Wu H, Bi Y, et al. Spatial and temporal distribution of falciparum malaria in China. Malar J. 2009;8:130.

67. Kulldorff M. SaTScan: Software for the spatial, temporal and space-time scan statistics. 1997.

68. Robertson C, Nelson TA. Review of software for space-time disease surveillance. Int J Health Geogr. 2010;9:16.

69. Aamodt G, Samuelsen SO, Skrondal A. A simulation study of three methods for detecting disease clusters. Int J Health Geogr. 2006;5:15.

\section{Submit your next manuscript to BioMed Central and we will help you at every step:}

- We accept pre-submission inquiries

- Our selector tool helps you to find the most relevant journal

- We provide round the clock customer support

- Convenient online submission

- Thorough peer review

- Inclusion in PubMed and all major indexing services

- Maximum visibility for your research

Submit your manuscript at www.biomedcentral.com/submit 